\title{
A 22-GHz MAP OF THE 30-DORADUS REGION
}

\author{
N.S. SABALISCK, Z. ABRAHAM \\ Instituto Astronomico e Geofisico \\ Universidade de Sao Paulo \\ Caixa Postal 30627 - CEP 01051 \\ Sao Paulo - SP \\ Brasil
}

ABSTRACT. We present a preliminary map of the 30-Doradus region at $22 \mathrm{GHz}$ with a HPBW of 4.6 arc. The results are compared with those from maps at lower frequencies and at similar resolution. Three supernova remnants were detected: N157b, MC 78 and MC 89.

\section{Observations}

The observations were made at $22 \mathrm{GHz}$ with the $13.7 \mathrm{~m}$ (radome enclosed) Itapetinga Radiotelescope in Brazil: the HPBW was 4'.6 arc. The receiver was a K-Band mixer with $1 \mathrm{GHz}$ d.s.b. bandwidth, a system temperature of $650 \mathrm{~K}$ and a sensitivity after $2 \mathrm{hrs}$ integration of $\sim 0.5$ Jy. It was operated in the total power mode. The map was made from scans in right ascension, separated by 2 ' arc in declination. The calibration was made on Virgo A, which has a flux density of $20.5 \mathrm{Jy}$ at $22 \mathrm{GHz}$ (Janssen et al. 1974). The S/T ratio is $58.6 \mathrm{Jy} / \mathrm{K}$.

\section{Results}

The sources detected in the 30-Dor map (Fig. 1) and their physical parameters are listed in Table 1.

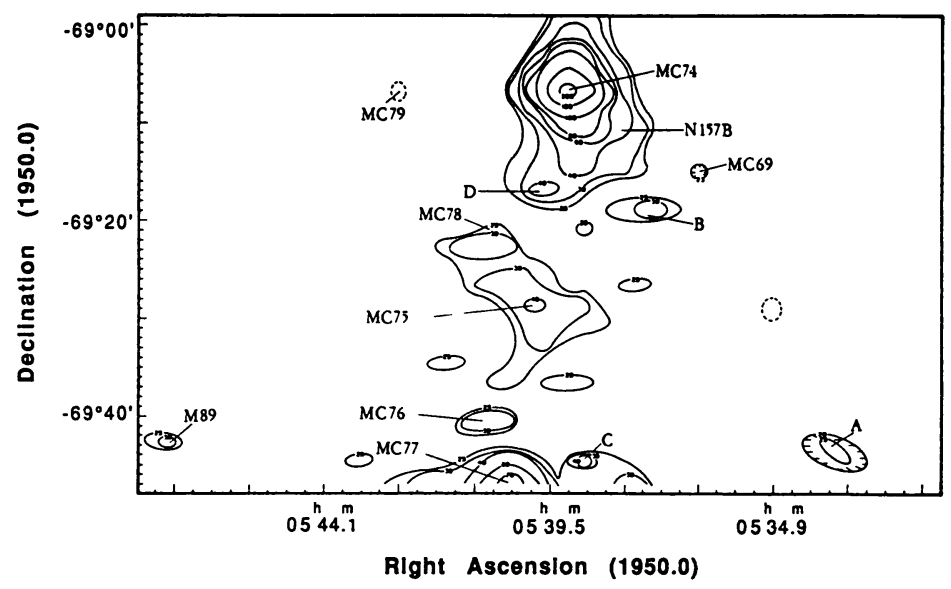

Figure 1. Radio continuum map at $22 \mathrm{GHz}$ of the 30-Dor region, with a resolution of 4'.6 arc. Contour levels are: $25,30,40,60,80,100,150 \& 200 \mathrm{mk}$ in antenna temperature. 
Table 1. $22 \mathrm{GHz}$ sources in the 30 -Dor region

\begin{tabular}{|c|c|c|c|c|c|c|c|c|}
\hline Source & $\begin{array}{r}\text { RA } \\
\mathbf{h} \mathbf{m} \mathbf{s} \\
\end{array}$ & $\begin{array}{l}\text { Dec } \\
\circ \quad \\
\end{array}$ & $\begin{array}{c}\text { Peak } \\
\text { temp. } \\
\text { mK }\end{array}$ & $\begin{array}{c}\text { Peak } \\
\text { flux } \\
\text { density } \\
\text { Jy }\end{array}$ & $\begin{array}{l}\text { Size } \\
\text { 'arc } \\
\end{array}$ & $\begin{array}{c}\text { Integ. } \\
\text { flux } \\
\text { density } \\
\text { Jy }\end{array}$ & Spectrum & Remarks \\
\hline $\mathbf{A}$ & 053342 & -6943.0 & 27 & 1.6 & 7.1 & 3.8 & $\mathbf{T}$ & \\
\hline MC 69 & 053624 & -6915.0 & 23 & 1.3 & point & & $\mathbf{T}$ & $30 \mathrm{Dor} \mathrm{CB} \dagger$ \\
\hline B & 053711 & -6919.0 & 33 & 1.9 & 4.8 & 2.0 & $\mathrm{~T}$ & \\
\hline N157B & 053807 & -6911.3 & 35 & 2.0 & - & - & NT & 30 Dor B* \\
\hline C & 053843 & -6945.0 & 42 & 2.5 & - & - & $\mathbf{T}$ & \\
\hline MC 74 & 053904 & -6907.5 & 207 & 12.1 & 6.7 & 26.9 & $\mathbf{T}$ & 30 Dor $A^{*}$ \\
\hline D & 053930 & -6917.0 & 41 & 2.4 & 7.3 & 6.1 & & \\
\hline MC 75 & 053953.4 & -6929.0 & 41 & 2.4 & 11.2 & 8.5 & $\mathbf{T}$ & $\mathrm{N} 158 \mathrm{C}$ \\
\hline MC 77 & 054024 & -6947.0 & 79 & 4.6 & & & $T$ & A 19, N159A \\
\hline MC 78 & 054039.5 & -6921.0 & 26 & 1.5 & - & - & NT & N158A \\
\hline MC 79 & 054232 & -6907.9 & & 0.5 & point & & $\mathrm{T}$ & \\
\hline MC 76 & 054020.8 & -6941.0 & 37 & 2.2 & $\cdot$ & $\cdot$ & $\mathrm{T}$ & $\mathrm{N} 160 \mathrm{~A}$ \\
\hline MC 89 & 054027.9 & -6943.0 & 31 & 1.8 & & & NT & \\
\hline
\end{tabular}

The sizes were derived assuming Gaussian models. Comparisons were made with the data from two other surveys at lower frequencies but at similar resolution (McGee et al. 1972; Clark et al. 1976) and the nature of the emission at $22 \mathrm{GHz}$ : Thermal (T) or non-thermal (NT), is indicated. The non-thermal sources 30 Dor B, MC 78 and MC 89, reported as plerionic supernova remnants (Milne et al. 1980, Long et al. 1981, Clark et al. 1982, Mathewson et al. 1983, Mills et al. 1984, Mills \& Turtle 1984) were all detected. The position of the maximum intensity in the source N158C coincides with the weaker component of two sources resolved in the $843 \mathrm{MHz}$ map of Mills \& Turtle (1984).

\section{Acknowledgment}

The work was partially supported by the Brazilian agency CNPq.

\section{References}

Clark, J.N., Little, A.G., Mills, B.Y. (1976). Aust. J. Phys. Astrophys.Suppl. no.40, 1.

Clark, J.N., Tuohy, I.R., Long, K.S., Szymkowiak, A.E., Dopita, M.A., Mathewson, D.S., Culhane, J.L. (1982), Astrophys. J. 255, 440.

Janssen, M.A., Golden, L.M., Welch, W.J. (1974), Astron. Astrophys. 33, 372.

Long, S.K., Helfand, D.J., Grabelsky, D.A. (1981), Astrophys. J. 248, 925.

Mathewson, D.S., Ford, V.L., Dopita, M.A., Tuohy, I.R., Long, K.S., Helfand, D.J. (1983), Astrophys. J. Suppl. 51, 345.

McGee, R.X., Brooks, J.W., Batchelor, R.A. (1972), Aust. J. Phys. 25, 581.

Mills, B.Y., Turtle, A.J., Little, A.G., Durdin, J.M. (1984), Aust. J. Phys. 37, 321.

Mills, B.Y. Turtle, A.J. (1984), IAU Symp. 108 Structure and Evolution of the Magellanic Clouds, S. van den Bergh and K.S. de Boer (eds.) (Reidel: Dordrecht) p. 283.

Milne, D.K., Caswell, J.L., Haynes, R.F. (1980), MNRAS 191, 469. 